Document downloaded from:

http://hdl.handle.net/10251/120675

This paper must be cited as:

Herranz Herruzo, Jl.; Valero-Nogueira, A.; Ferrando Rocher, M.; Bernardo Clemente, B.; Vila Jiménez, A.; Lenormand, R. (2018). Low-Cost Ka-band Switchable RHCP/LHCP Antenna Array for Mobile SATCOM Terminal. IEEE Transactions on Antennas and Propagation. 66(5):2661-2666. https://doi.org/10.1109/TAP.2018.2806421

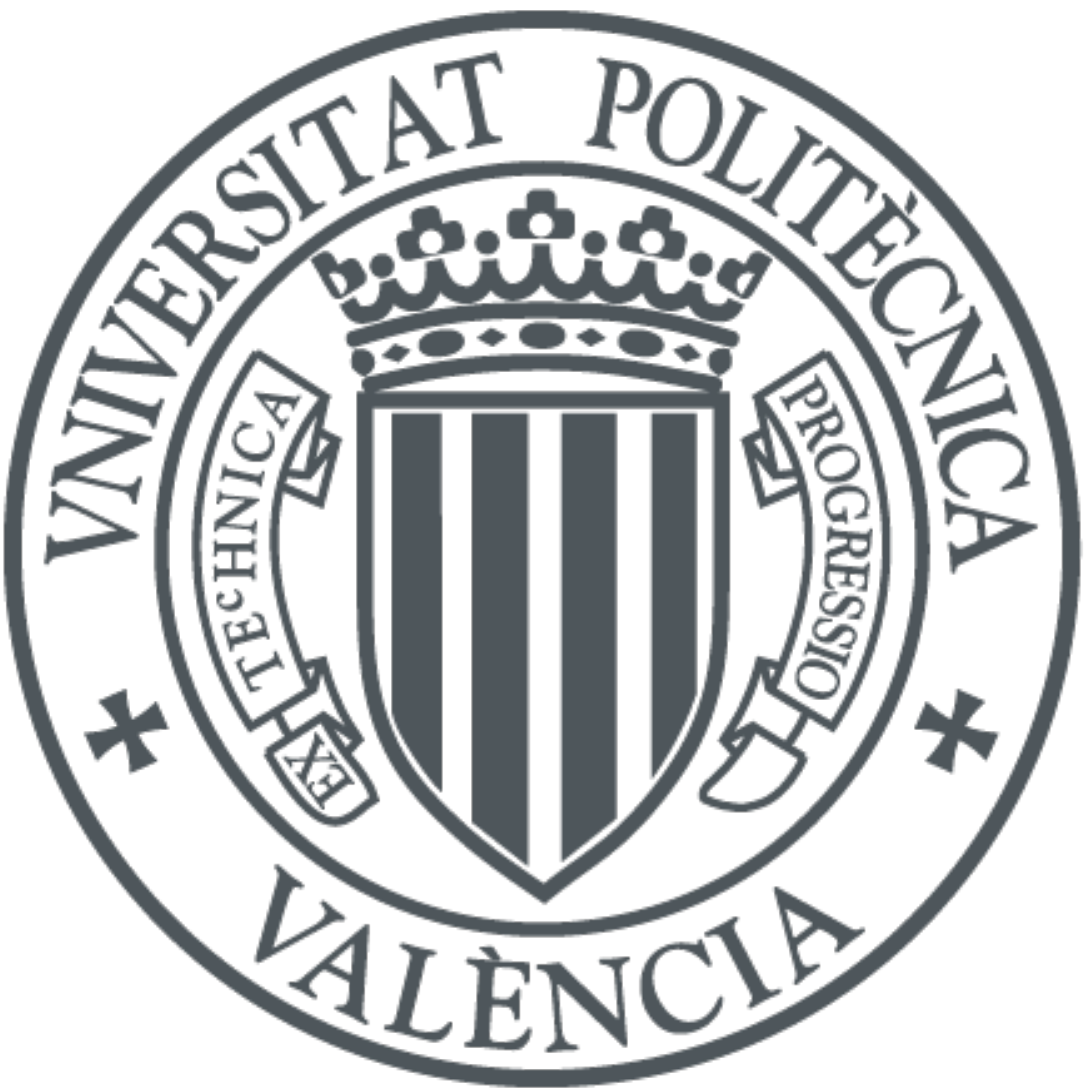

The final publication is available at

http://doi.org/10.1109/TAP.2018.2806421

Copyright Institute of Electrical and Electronics Engineers

Additional Information

(c) 2018 IEEE. Personal use of this material is permitted. Permission from IEEE must be obtained for all other users, including reprinting/ republishing this 


\title{
Communication Low-cost Ka-band Switchable RHCP/LHCP Antenna Array for Mobile SATCOM Terminal
}

\author{
Jose I. Herranz-Herruzo, Member, IEEE, Alejandro Valero-Nogueira, Senior Member, IEEE, \\ Miguel Ferrando-Rocher, Bernardo Bernardo, Antonio Vila and Régis Lenormand
}

\begin{abstract}
Achieving a functional antenna for mobile SATCOM terminals in Ka band is probably one of the most challenging tasks in current antenna engineering. Even more if this terminal has to exhibit a low profile and be "affordable". This quest is involving many companies in the field. Our contribution represents one of such efforts. The antenna is based on slotted waveguide array technology to maximize efficiency and it features a number of novel solutions, going from its robust polarization switching mechanism, to the use of a thin wideband polarizer and the utilization of groove gap waveguides. This paper reports the measured data of a fully functional prototype to validate its novel contributions.
\end{abstract}

Index Terms-mobile satellite communications, slotted waveguide arrays, circular polarization.

\section{INTRODUCTION}

Satellite communications are evolving to meet growing market needs on broadband applications. Many satellite operators are migrating to cellular Ka-band systems, increasing throughput speeds in at least one order of magnitude compared to current Ku-band systems [1]. Multi-spot coverage poses challenging problems to mobile terminals, where one of the most critical points remains the implementation of reliable antennas matching current regulations and able to automatically switch polarization for cell handover. Furthermore, the demand for limited-volume RX-TX antennas compatible with required $G / T$ values puts the focus on scannable planar array antennas. Even though steerable array antennas, either fully mechanical or hybrid electronic-mechanical, are a very mature technology, available in the Ku-band market, its implementation in Ka band is not at all straightforward. Phase shifter MMIC technology is still very lossy and expensive for most mobile terminals in civil applications. Besides, the additional implementation of the circular polarization switch subsystem calls for a dedicated design which has barely been attempted in the past, even for $\mathrm{X}$ or Ku-band antennas.

In this regard, satisfactory designs enjoying TX/RX operation, dually polarized, low profile and low cost antennas for SATCOM on the move (SOTM) in Ka band, are scarce. Recently, European Space Agency (ESA) granted project LOCOMO (Low cost and compact Ka-band mobile SATCOM terminal) to develop a Ka-band SOTM terminal, able to be integrated onto terrestrial vehicles [2]. Such equipment, shown in Fig. 1, would provide broadband services anywhere and anytime, even in motion, at a reasonable cost. The target applications are national security, emergency and dual-use systems, where military frequency bands will be made available for civilian missions. The challenge of LOCOMO terminal is to reach a commitment of low profile and low cost while complying with satellite regulations [3] and high performance services.

This work was supported by the European Space Agency under contract 400010860913NLUS - project LOCOMO.

The first four authors are with the Instituto de Telecomunicaciones y Aplicaciones Multimedia, Universitat Politcnica de Valncia, Valencia 46022, Spain (e-mail: jiherhe@upvnet.upv.es; avalero@dcom.upv.es; miguelferrandorocher@gmail.com; berbercl@upvnet.upv.es; anviji@upvnet.upv.es). The last author is a private consultant (e-mail: lenormand.reg@outlook.fr).

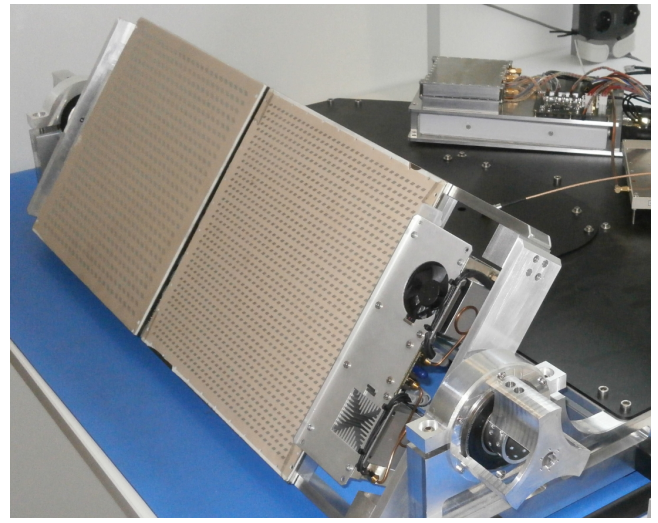

Fig. 1. LOCOMO antenna mounted on rotatable axis. Two antenna subpanels for transmit and receive are visible. Two more are on the backside.

The terminal is composed of three main subsystems: Antenna, Radiofrequency and PAT (Pointing-Acquisition-Tracking). This paper will be devoted only to the antenna subsystem, stressing those new features that have not been attempted in the field so far. With that purpose, next section details the specifications imposed, describes the overall antenna architecture and the technological solutions chosen. Section III summarizes the analysis and optimization approaches followed. Assembly and measurements of full-size transmit and receive antennas are reported in Section IV.

\section{Antenna Specifications And ARChitecture}

The LOCOMO terminal is intended to provide broadband satellite communications on mobile ground vehicles. For the sake of brevity, only those specifications relevant for the antenna subsystem will be detailed: transmit (TX) and receive (RX) bands are $29.5-31 \mathrm{GHz}$ and $19.7-21.2 \mathrm{GHz}$, respectively. Antenna gain should be greater than $36 \mathrm{dBi}$ for transmit and $34 \mathrm{dBi}$ for receive. Patterns must comply with ETSI regulations [3]. Polarization is circular and switchable, with a target axial ratio (AR) better than $2 \mathrm{~dB}$. The antenna coverage spans $360^{\circ}$ in azimuth and from $5^{\circ}$ to $90^{\circ}$ in elevation. Finally, the antenna must keep a low profile, being $30 \mathrm{~cm}$ the maximum height.

The solution adopted, shown in Fig. 1, implements a mechanical antenna pointing. The polarization switching resorts to a mechanical axis as well. The rotatable structure exhibits two faces, front and back, so that polarization is switched by a complete $180^{\circ}$ rotation around its major axis [4]. Previous works on this issue performed the switching through a lateral displacement of a polarization layer [5], [6]. Those approaches, however, lack robustness during switching and may lead to poorer AR performance. Our solution comprises four subpanels, two at the front side (TX-RHCP and RX-LHCP) and two more at the backside (TX-LHCP and RX-RHCP). Despite such double scheme certainly increases thickness and somehow complexity, these dedicated antennas provide better electrical performance. 


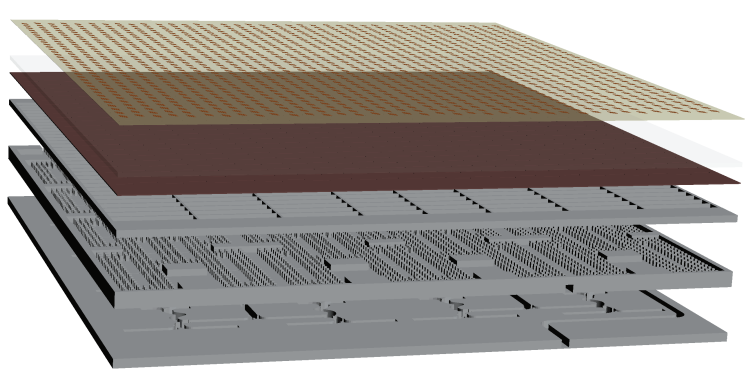

Fig. 2. 3D model of the TX antenna subpanel.

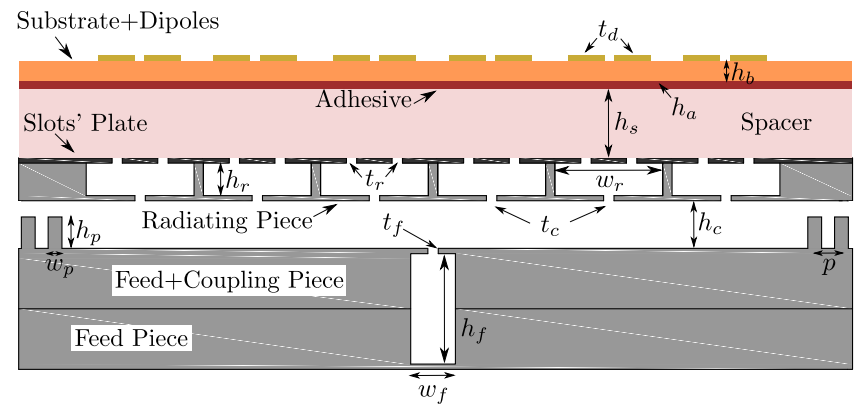

Fig. 3. Schematic side view of antenna assembly.

All four antennas share a common architecture based on full-metal slotted waveguide array technology to minimize losses. Figs. 2 and 3 depict respectively the 3D model and the schematic side view of one subpanel. Four layers can be identified: going from top to bottom, these are polarizer, radiating layer, coupling layer and feed layer.

The polarizing layer is made up of an array of printed dipoles. This concept was tested already in [7], where it was proved that a wide polarization bandwidth can be achieved by placing three tilted parasitic dipoles above each slot. In a practical implementation, these dipoles are etched on a very thin substrate which rests on top of a foam spacer providing the required height. Spacer relative permittivity should be as close to one as possible to relax mutual coupling effects and not compromise bandwidth. More details about dipoles' design can be read in [7] and will be omitted here.

The radiating layer consists of rectangular waveguides with resonant shunt slot arrays. The radiating aperture is subdivided into smaller subarrays to improve operation bandwidth. Coupling layer below is responsible for connecting feed network to the radiating waveguides forming each subarray. For such task, rectangular waveguides are conventionally used. However, from a fabrication point of view, the joints between these two layers introduce serious challenges since imperfect metal contact leads to important loss due to leakage. Therefore a good deal of screws are usually employed to assess shielding, and very often there is no room for all of them.

To overcome this problem, the antenna utilizes a new type of waveguide known as Groove Gap waveguide [8] at the coupling layer. As shown in Figs. 2 and 3, waveguide sidewalls are replaced by several rows of nails, leaving a small gap between them and the upper wall. Nails create a high-impedance condition such that fields remain confined within the groove without resorting to any closed shielding. The fundamental mode propagating along such groove reveals a field distribution surprisingly close to that of a rectangular waveguide. In Fig. 4, propagation constant for the fundamental mode of a groove gap waveguide (GGW) is compared to that of a rectangular waveguide (RWG) having the same cross-section. The RX band is the targeted one here. Although curves manifest a slightly different slope, they can be forced to be identical for a given frequency just by adjusting

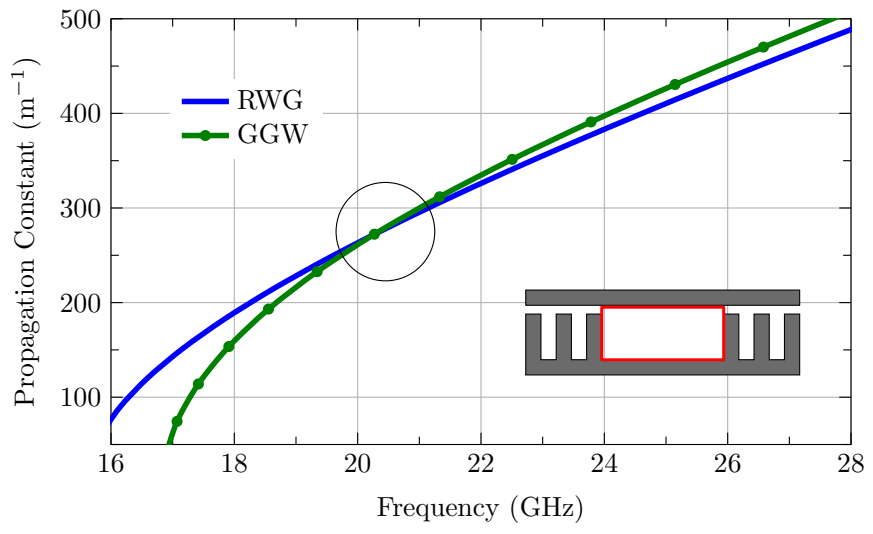

Fig. 4. Propagation constant of a rectangular waveguide with cross-section $9.62 \times 4.6 \mathrm{~mm}^{2}$ (red box in inset), and an equivalent Groove Gap waveguide. Nails are $2 \mathrm{~mm}$ wide and $3.6 \mathrm{~mm}$ high. Nail periodicity is $5 \mathrm{~mm}$.

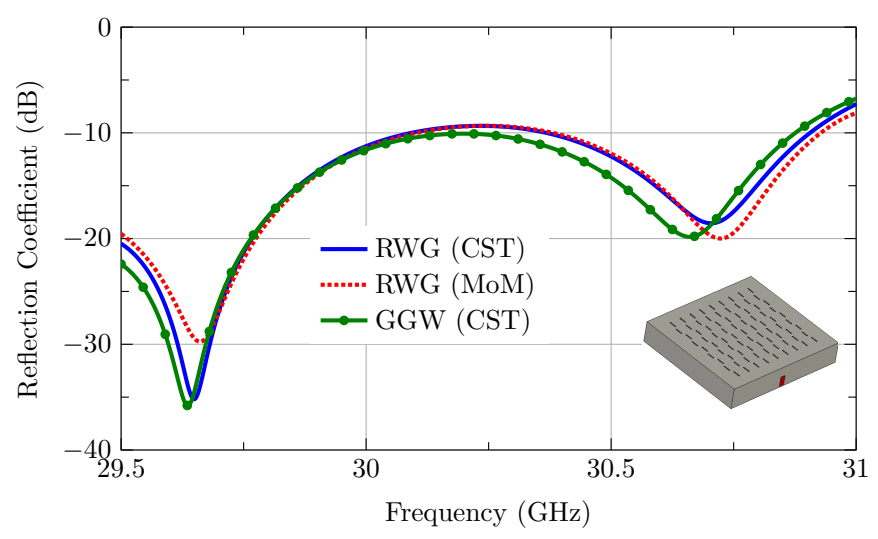

Fig. 5. Reflection coefficient comparison for a subarray taken from TX antenna.

nails width and periodicity.

For the sake of illustration, let us consider a subarray taken from TX antenna. The subarray is highlighted in Fig. 11. It has been simulated separately considering both types of waveguides at the coupling layer, rectangular and groove gap. Parasitic dipoles have been removed from simulation for simplicity. Antenna key parameters, such as reflection coefficient and radiation pattern, are compared in Figs. 5 and 6, respectively. The whole band exhibits the same level of adaptation and just a slight shift in frequency can be observed. Very encouraging results can be viewed also for the E-plane radiation pattern. Lastly, the effectiveness of nails in field confinement is evidenced in Fig. 7, where the electric field distribution is shown within the groove waveguides. Hence, it can be concluded that relying on a rectangular waveguide for fast analysis and substituting it by an equivalent groove gap waveguide for fabrication is not a complete leap in the dark. However, such equivalence can be assured only within a moderate bandwidth around the design frequency. This fact will be addressed in Section IV with experimental data.

Finally, antenna's bottom layer consists of a corporate E-plane feeding network. Despite E-plane selection leads to a thicker panel, splitters generally show wider bandwidth than their H-plane counterparts. Moreover, the narrower E-plane groove enables a single-layer network, even for the high number of outputs needed in TX antenna. One last, but not minor, advantage of E-plane circuits is the fact that electric shielding can be readily achieved by fabricating them in two symmetric halves. This technique leverages the electric current 


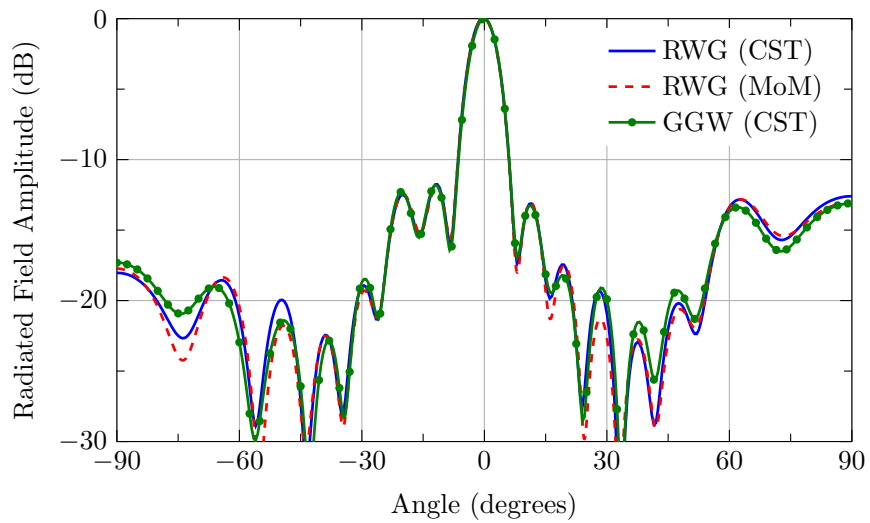

Fig. 6. E-plane radiation pattern comparison at $30.25 \mathrm{GHz}$ for a subarray taken from TX antenna.
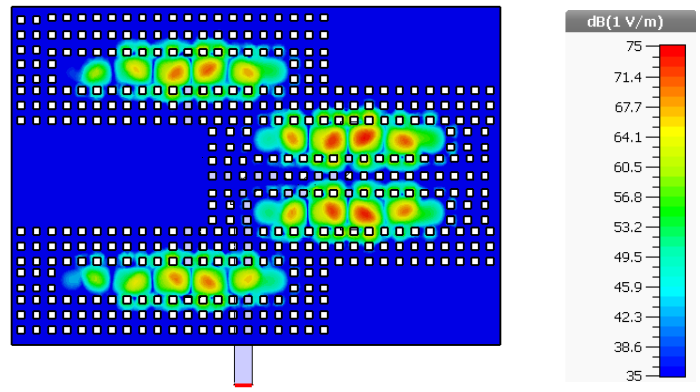

Fig. 7. Electric field amplitude distribution (dB) within the coupling waveguides in a subarray taken from TX antenna. Input port from the feed network is at a lower level, highlighted in red.

null along broad-wall waveguide axis. Note that using groove gap waveguides at this feeding layer, though feasible, would complicate splitters design and fabrication.

\section{ANALYSIS AND OPTIMIZATION}

Design of large array antennas cannot be faced using commercial codes only. The present work relies on an accurate hybrid analysis technique which combines an in-house Method of Moments (MoM) code [9] and CST Microwave Studio [10]. Our own MoM code reaches up to the subarray inputs at the feed layer whereas CST software simulates the whole E-plane feed network. Both results are connected through their corresponding S-parameter matrices, achieving a full electromagnetic description of the antenna. With the aim of accrediting its accuracy, the method is compared with one measured sample of the fabricated antenna. Fig. 8 plots the radiation pattern of RX antenna at the centre frequency. The comparison between simulated and measured patterns advances the remarkable accuracy of the developed analysis. Further results concerning the matching parameters will be displayed in Section IV.

Now, such accurate analysis is the base for an efficient optimization method which will provide the complete description of the antenna layout. The approach implemented is an iterative correction process starting from an initial design based on classical circuital equivalence [9]. Given the antenna architecture, the optimization follows a downward multilevel strategy, i.e. from radiating to feeding layers, converging in just a few iterations. The correction approach at each individual layer has been adapted from a previous work [11]. This method plays a crucial role since we are dealing with strong mutual coupling among slots [9].

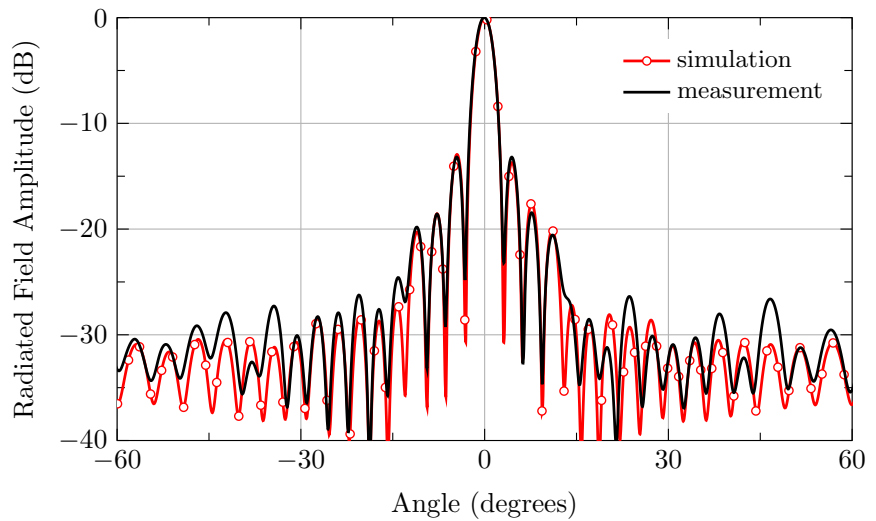

Fig. 8. Comparison of measured and simulated pattern in $\phi=0^{\circ}$ cut for the $\mathrm{RX}$ antenna at the center frequency, $20.45 \mathrm{GHz}$.

Lastly, antenna design has dealt with a major challenge of resonanttype series-fed arrays, which is its narrow-band performance. Note that RX antenna must comply with a moderate bandwidth around 7.5\%. Radiation pattern stability, on the one hand, has been reached by properly subarraying the panel, alleviating the well-known long waveguide effect. The specified matching level in the whole band, on the other hand, has been forced by applying the so-called detuning techniques. A careful implementation of this approach provides a noticeably increase in matching bandwidth without an apparent sacrifice in radiation performance.

\section{EXPERIMENTAL RESULTS}

\section{A. Antenna Assembly}

The four subpanels being part of the SATCOM terminal were fully constructed in-house, except for the dipoles sheet, which was supplied by an external provider. The same fabrication and assembly process has been employed for every subpanel, seen in Figs. 2 and 3. Each metallic piece has been fabricated in aluminum type AL7075 using Datron M25 CNC milling machine.

Going from bottom up, the feeding network has been split along the E-plane symmetry plane. Note that matching faces have to be treated to show good enough flatness. The upper block, named in Fig. 3 as Feed +Coupling piece, contains also the coupling layer on its upper face, where nails are milled to shape the groove gap waveguides. The radiating layer is set on top of the coupling layer. Slots are milled on a separate aluminum sheet which is soldered to the radiating waveguides piece. Therefore, these two pieces were first silver-plated to accommodate a soldering process based on conducting paste. With the aid of a stencil and an ad-hoc device for proper alignment, the paste was applied as depicted in Fig. 9. Later, radiating waveguides and slots plate were soldered together by curing the paste in an oven under a specified temperature profile.

The polarizing dipoles layer is realized on a thin PCB sheet, namely a Neltec NY92200, 127 microns in thickness. This PCB is bonded to a Rohacell HF51 foam spacer by an adhesive sheet, commercially sold as Cool-Bond CB7130. Bonding is done by applying minimal pressure at a stable temperature around $100^{\circ} \mathrm{C}$. Finally, the foam is also bonded to the slots plate to avoid undesired air gaps that might greatly affect polarization performance.

Each pair of antennas provided similar measured results, which demonstrates the repeatability of the process chosen. In the next, these measurements for two units only, one receive and one transmit, are shown and discussed. 


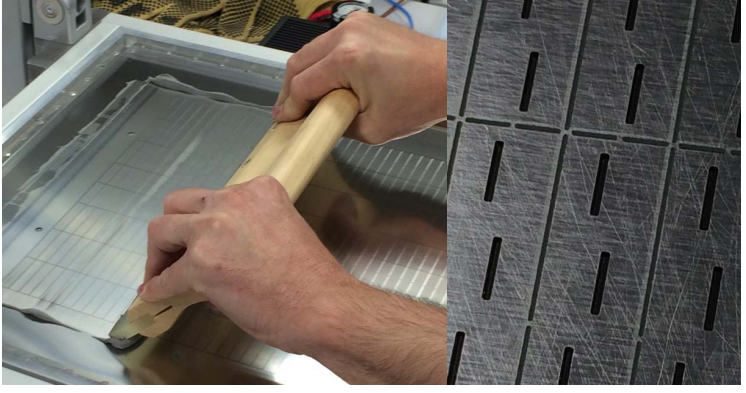

Fig. 9. The soldering paste is set in place manually with the aid of a stencil. The picture to the right shows the outcome of the process. The black lines framing the slots is the deposited paste.

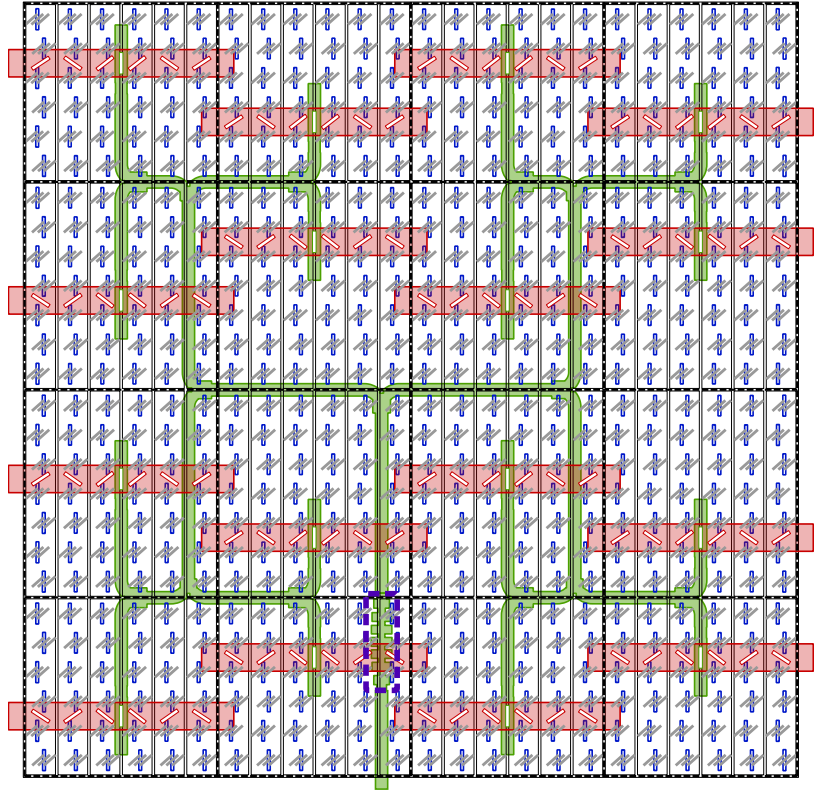

Fig. 10. Layout of RX antenna comprising $4 \times 4$ subarrays. It shows parasitic dipoles (grey), radiating slots (blue), radiating waveguides (black), coupling waveguides (red) and feed network (green). Low-pass filter is boxed in dashed purple line.

\section{B. Experimental results}

An aperture size of $27 \times 27 \mathrm{~cm}^{2}$ is chosen for both RX and TX subpanels to achieve the specified gain while satisfying the height constraint. However, while RX aperture intends to maximize the received signal by adopting a uniform illumination, the TX subpanel requires a tapered distribution to comply with regulation mask [3]. In this case, a 23-dB Taylor one-parameter distribution was targeted for both planes. For that same reason, preservation of low-sidelobe radiation patterns in the whole TX band compels an intensive $8 \times 8$ subarray subdivision. A looser $4 \times 4$ arrangement is enough for the RX subpanel instead. Array arrangement encompasses $26 \times 24$ and $38 \times 34$ radiating elements for RX and TX antennas, respectively. Each element comprises one slot and three dipoles.

The complete antenna layouts are shown in Figs. 10 and 11 for RX and TX subpanels, respectively. A color scheme has been used to help visualization. Thicker black dashed lines are introduced to delimit the extent of each subarray. For the sake of completeness, Table I collects the main dimensions for each layer, as defined in Fig. 3. As a general rule, letters $h, w, l$ and $t$ denote height, width, length and thickness of each element, respectively. Slots and dipoles dimensions at every layer are individually adjusted by the optimization process.

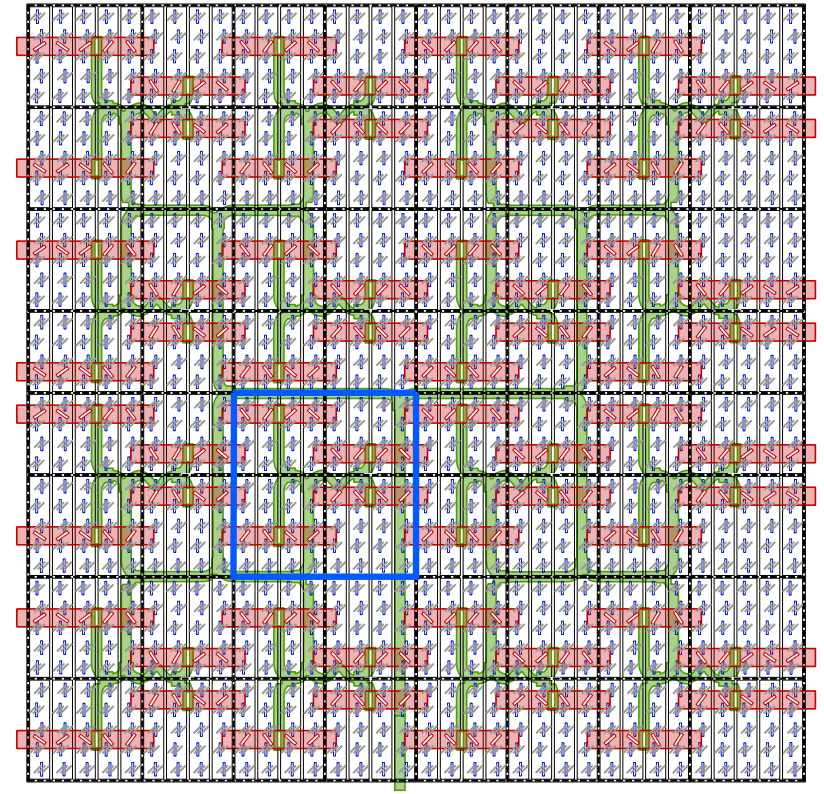

Fig. 11. Layout of TX antenna comprising $8 \times 8$ subarrays. It shows parasitic dipoles (black), radiating slots (blue), radiating waveguides (black), coupling waveguides (red) and feed network (green).

TABLE I

GEOMETRICAL DIMENSIONS (MM) OF RX AND TX ANTENNAS.

\begin{tabular}{|c|c|c||c|c||c|c||c|c|}
\hline \multicolumn{7}{|c|}{ Feed Layer } \\
\hline RX & $h_{f}$ & 10.668 & $w_{f}$ & 4.318 & $l_{f}$ & - & $t_{f}$ & 0.5 \\
\hline TX & $h_{f}$ & 7.112 & $w_{f}$ & 3.556 & $l_{f}$ & - & $t_{f}$ & 0.5 \\
\hline \hline \multicolumn{8}{|c|}{ Coupling Layer } \\
\hline \multirow{2}{*}{ RX } & $h_{c}$ & 4.6 & $w_{c}$ & 9.619 & $l_{c}$ & 79.24 & $t_{c}$ & 0.5 \\
\cline { 2 - 9 } & $h_{p}$ & 3.6 & $w_{p}$ & 2.0 & $p$ & 5.0 & - & - \\
\hline \multirow{2}{*}{ TX } & $h_{c}$ & 3 & $w_{c}$ & 6.302 & $l_{c}$ & $40.1-48.1$ & $t_{c}$ & 0.5 \\
\cline { 2 - 9 } & $h_{p}$ & 2.5 & $w_{p}$ & 1.5 & $p$ & 3.1 & - & - \\
\hline \hline \multicolumn{8}{|c|}{ Radiating Layer } \\
\hline RX & $h_{r}$ & 3.126 & $w_{r}$ & 10.420 & $l_{r}$ & $61.9-72.2$ & $t_{r}$ & 0.5 \\
\hline TX & $h_{r}$ & 2.644 & $w_{r}$ & 7.050 & $l_{r}$ & $34.8-27.9$ & $t_{r}$ & 0.5 \\
\hline \hline \multicolumn{8}{|c||}{ Multilayered Polarizer } \\
\hline RX & $h_{s}$ & 3.0 & $h_{a}$ & 0.076 & $h_{b}$ & 0.127 & $t_{d}$ & 0.018 \\
\hline TX & $h_{s}$ & 2.1 & $h_{a}$ & 0.076 & $h_{b}$ & 0.127 & $t_{d}$ & 0.018 \\
\hline
\end{tabular}

As can be seen in layout sketches, coupling waveguides are not placed at the center of the radiating waveguides, as usually done. They have had to be kept two rows apart from each other to accommodate a few rows of nails between them for effective isolation, as it was demonstrated in Fig. 7. Those nails surrounding every coupling waveguide are omitted in Figs. 10 and 11 for clarity. Note that the nail pattern, as seen in Fig. 7, leaves the same room originally occupied by the depicted coupling waveguides.

The feeding network adopts a conventional E-plane corporate topology. Smooth chamfered splitters have been optimized to achieve a wideband return-loss performance. In contrast to the 1-to-16 RX network, whose uniform power sharing facilitates its design, the more intricate 1-to-64 TX network demands a computationally more demanding optimization. Note that the tapered distribution requires an individual optimization of every unbalanced splitter.

A side effect of the aforementioned displacement of the coupling waveguides is that it forces networks outputs to be arranged on a nonregular grid. Therefore, special care should also be given to phase equalization. As can be observed in RX layout, the different lengths of output branches are compensated by a lateral displacement of the penultimate divider. Such path equalization restores wideband in- 


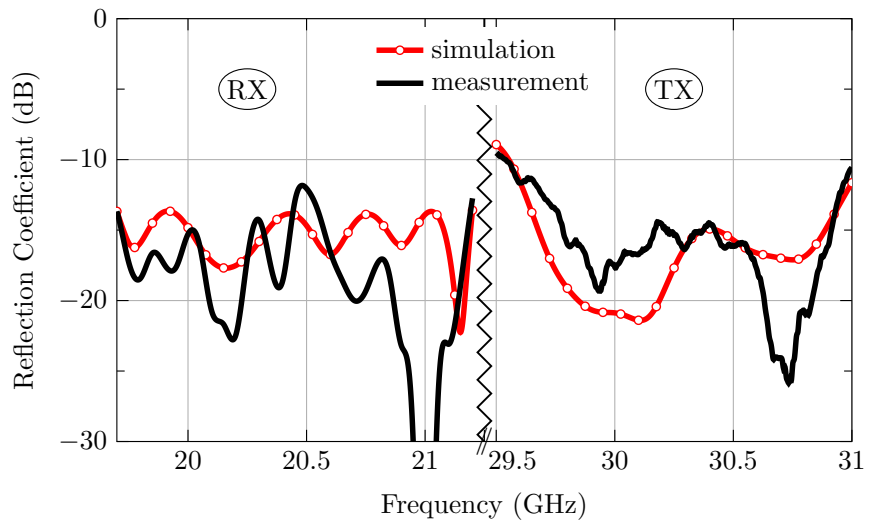

Fig. 12. Measured $S_{11}$-parameter for RX and TX subpanels. Simulated values are also shown for comparison. Frequency axis is broken to help visualization.

phase distribution, an imperative feature to meet the specified antenna performance. Such concern is aggravated for the TX network. As one can realize in Fig. 11, smaller subarrays force feed points to be very close to each other, to such extent that the splitter displacement is not enough to achieve equal path lengths. The additional phase hop needed is attained by smooth meandered sections carefully optimized.

As a final remark, a multi-stage waveguide low-pass filter has been designed and seamlessly integrated at RX antenna input to fulfill the system requirement on TX band rejection.

Now, the most relevant experimental outcomes are reported in order to assess the specification compliance over both bands. Input matching performance compared with simulation is shown in Fig. 12. Reflection coefficient fulfills the $-10 \mathrm{~dB}$ specification, remaining even below $-15 \mathrm{~dB}$ for a wide frequency range in both bands. The detuning technique employed turned out to be key to reach this specification. A very satisfactory agreement with simulation can be noticed, revealing the reliability of the analysis performed.

Figs. 13 and 14 plot the radiation patterns for RX and TX subpanels, respectively. Both main cuts at five equispaced frequencies within each band are shown. In order to assess the compliance with regulation, the TX graphs include the current ETSI template and renormalize the measured patterns to a maximum transmitted EIRP of $20 \mathrm{dBW} / 40 \mathrm{kHz}$. For both antennas, radiation patterns exhibit the expected shape. Particularly remarkable is the pattern stability with frequency along the elevation plane. The azimuth cut, on the other hand, shows a noticeable deterioration at both band edges, particularly at the upper frequency. This effect can be attributed to the wellknown stronger mutual coupling along the slots' E-plane, aggravated here by the polarizer. Another plausible cause recalls the equivalence between groove gap and rectangular waveguides, outlined in Fig. 4. The slight difference in propagation constant at band edges, will affect the coupling slots excitation mainly, which in turn has an impact on the elevation pattern. As a consequence, it should be admitted that we are close or even beyond the bandwidth limits of the presumed equivalence between these waveguides. A deeper investigation on improvements of this equivalent model is needed, though it is left for future work.

Gain and axial ratio largely determine antenna subsystem performance. These values have been measured accurately for broadside maximum in a certified compact-range test facility and are sampled in Table II at five test frequencies in both bands. The demanding specification of polarization purity is broadly satisfied, since a worstcase axial ratio of $1.3 \mathrm{~dB}$ is measured in $\mathrm{RX}$ band and $1.22 \mathrm{~dB}$ in TX band. Already demonstrated in [7], the three-dipole solution features an extremely compact wideband polarizer, particularly suitable for

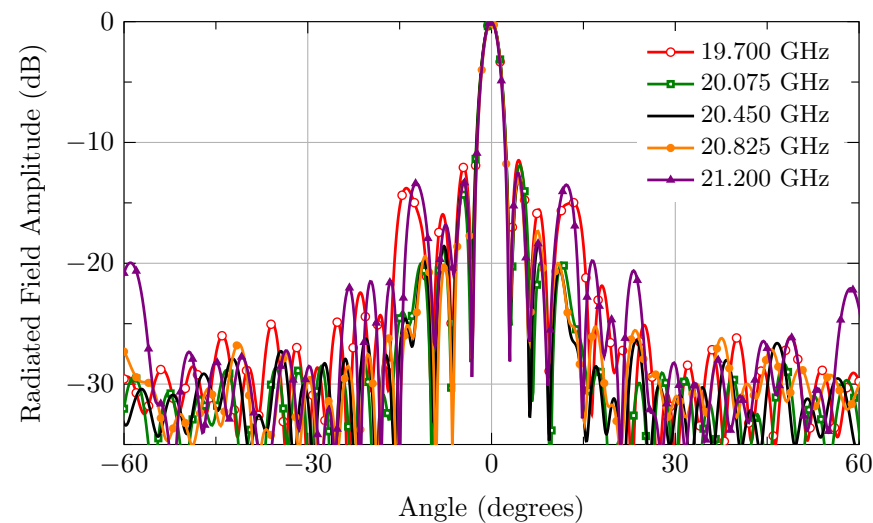

(a) Azimuth plane $\left(\phi=0^{\circ}\right)$

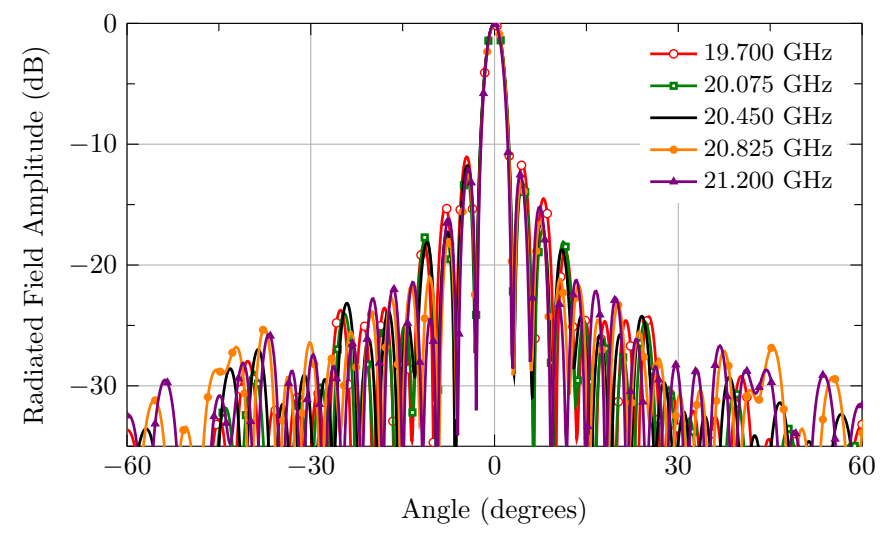

(b) Elevation plane $\left(\phi=90^{\circ}\right)$

Fig. 13. Measured patterns of RX subpanel at five equispaced frequencies.

TABLE II

MEASURED PERFORMANCE OF RX AND TX ANTENNAS.

\begin{tabular}{|l|c|c|c|c|c|}
\hline \multicolumn{7}{|c|}{ RX Antenna } \\
\hline Frequency (GHz) & $\mathbf{1 9 . 7}$ & $\mathbf{2 0 . 0 7 5}$ & $\mathbf{2 0 . 4 5}$ & $\mathbf{2 0 . 8 2 5}$ & $\mathbf{2 1 . 2}$ \\
\hline \hline Axial Ratio (dB) & 1.31 & 0.75 & 0.27 & 0.35 & 0.89 \\
\hline \hline Directivity (dBi) & 35.04 & 35.77 & 35.83 & 35.91 & 35.27 \\
\hline Illumitation eff. (\%) & 85.9 & 97.9 & 95.4 & 93.9 & 78.2 \\
\hline \hline Realized Gain (dBi) & 33.53 & 34.77 & 34.56 & 34.59 & 33.49 \\
\hline Radiation eff. (\%) & 73.8 & 80.6 & 78.6 & 75.3 & 70.1 \\
\hline \hline \multicolumn{7}{|c|}{ TX Antenna } \\
\hline Frequency (GHz) & $\mathbf{2 9 . 5}$ & $\mathbf{2 9 . 8 7 5}$ & $\mathbf{3 0 . 2 5}$ & $\mathbf{3 0 . 6 2 5}$ & $\mathbf{3 1 . 0}$ \\
\hline \hline Axial Ratio (dB) & 0.56 & 0.38 & 0.07 & 0.36 & 1.22 \\
\hline \hline Directivity (dBi) & 38.09 & 38.13 & 38.43 & 38.74 & 38.32 \\
\hline Illumitation eff. (\%) & 71.8 & 70.6 & 73.8 & 77.3 & 68.5 \\
\hline \hline Realized Gain (dBi) & 35.78 & 36.84 & 36.87 & 37.44 & 36.44 \\
\hline Radiation eff. (\%) & 66.2 & 75.9 & 71.9 & 75.0 & 71.2 \\
\hline
\end{tabular}

SOTM applications. Table II also displays Directivity and Aperture Illumination Efficiency. Directivity exhibits a peak value of $35.9 \mathrm{dBi}$ for RX antenna and $38.7 \mathrm{dBi}$ for TX antenna, corresponding to an illumination efficiency of $94 \%$ and $77 \%$, respectively. Such values agree well with the synthesized aperture distribution. It should be stressed that the 1-dB-drop Directivity bandwidth contains both targeted RX and TX bands.

Table II shows the measured Realized Gain and Loss Efficiency as well. Given its relevance, the Realized Gain has been plotted in Fig. 15 for the whole bands as well. A very stable gain frequency response confirms the suitability of the proposed solution for receiving and transmitting operation. A peak gain of $34.96 \mathrm{dBi}$ can be read in 


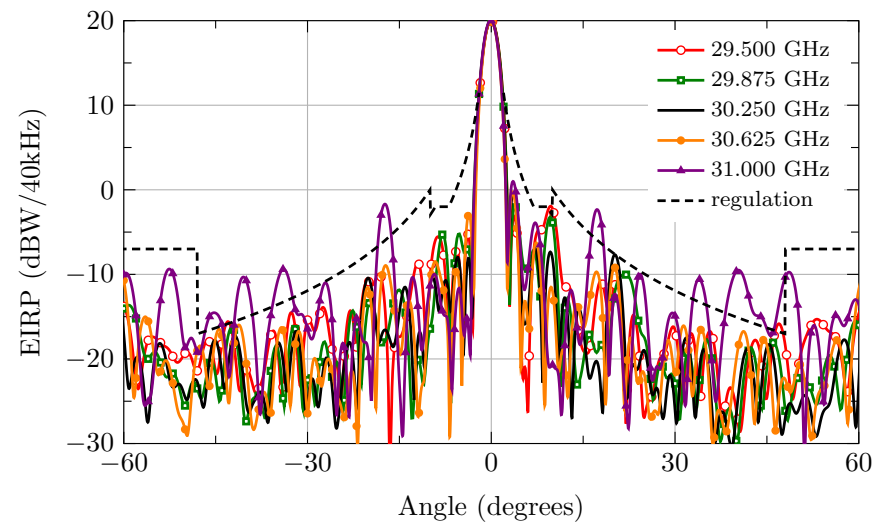

(a) Azimuth plane $\left(\phi=0^{\circ}\right)$

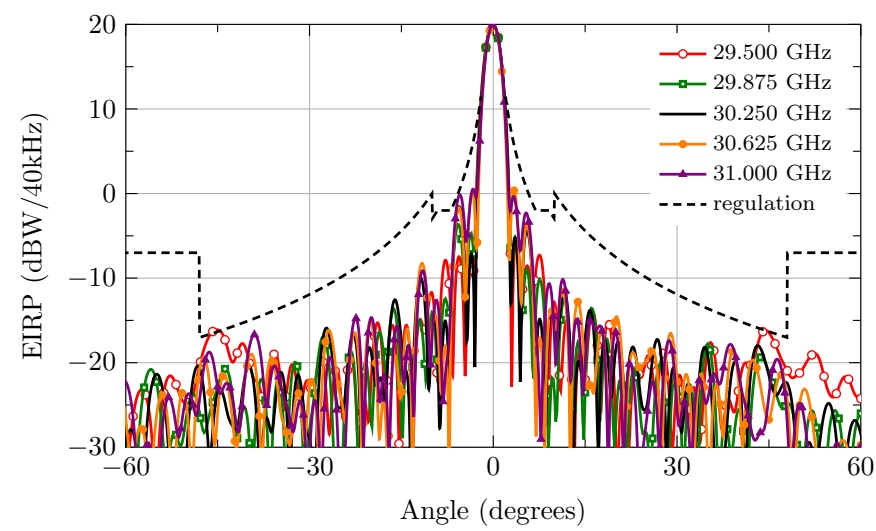

(b) Elevation plane $\left(\phi=90^{\circ}\right)$

Fig. 14. Measured EIRP density patterns of TX subpanel at five equispaced frequencies. Corresponding ETSI template is also shown for reference.

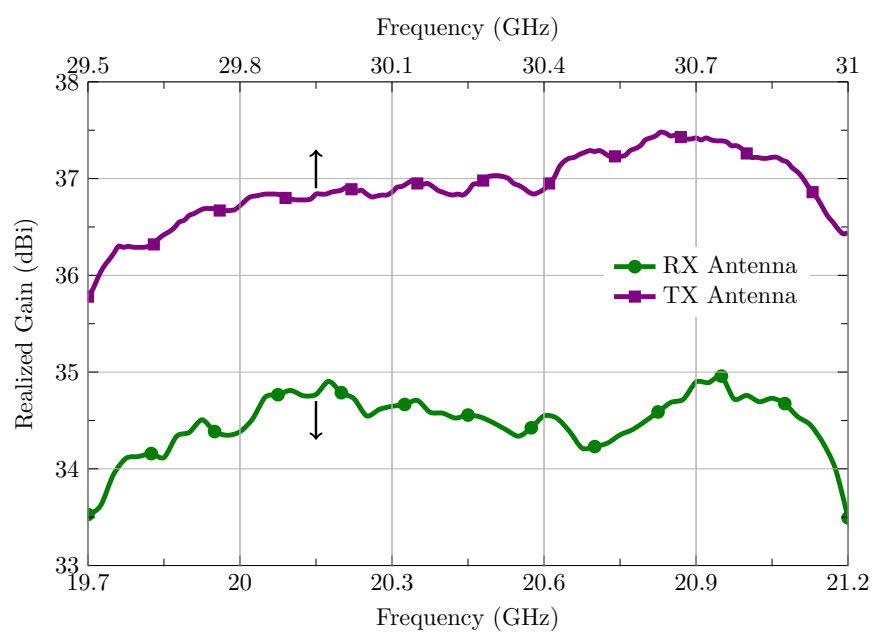

Fig. 15. Realized gain vs. frequency for RX and TX subpanels.

the RX band and $37.48 \mathrm{dBi}$ in the TX band. Only at the very end of each band, gain drops as it would do outside its operation band due to the reasons discussed above. However, zoomed inspection of Fig. 15 restricts such anomaly to the last $20 \mathrm{MHz}$ in both bands.

Lastly, Radiation Efficiency figures shown in Table II hold very decent values, with sampled maxima around $81 \%$ and $76 \%$ for RX and TX subpanels, respectively. After a loss budget assessment by simulation for the TX band, those 1.2- $\mathrm{dB}$ losses can be roughly split into $0.6 \mathrm{~dB}$ attributed to the multilayered polarizer and $0.5 \mathrm{~dB}$ to the feed network. The remaining losses, around $0.1 \mathrm{~dB}$, would correspond to both coupling and radiating waveguide layers. It has certainly been evidenced the effectiveness of the solutions adopted to prevent field leakage: the groove-gap waveguiding technology in coupling layer and the divided-in-half E-plane waveguide in feeding network. These contributions turned out to be fundamental to attain a successful solution.

\section{CONCLUSion}

This paper describes the antenna solution adopted for a SATCOM on the move ground terminal in Ka band. The antenna was developed in the framework of project LOCOMO. The antenna architecture reproduces a traditional slotted waveguide array, comprising radiating and coupling layers and a feed network. However, the adopted solution incorporates a number of novel features which turned out to provide capital improvements in terms of low profile, efficiency, pattern stability and polarization bandwidth. These are the threedipole polarizer, the groove gap waveguide at the coupling layer, and the use of a single-layer E-plane corporate-feed network. Implicitly, the successful experimental results have pointed out the accuracy of the hybrid analysis approach and the care taken in the inhouse antenna fabrication. All these features jointly contributed to a remarkable performance.

\section{REFERENCES}

[1] E. Feltrin and E. Weller, "New frontiers for the mobile satellite interactive services," in Advanced satellite multimedia systems conference (asma) and the 11th signal processing for space communications workshop (spsc), 2010 5th. IEEE, 2010, pp. 155-161.

[2] M. J. González, A. Pellón, R. Lenormand, A. Hirsch, A. ValeroNogueira, J. I. Herranz-Herruzo, J. Petit, Y. Haentjens, N. Capet, E. Sáenz et al., "Low cost and compact Ka-band mobile Satcom terminal," in Antennas and Propagation (EuCAP), 2014 8th European Conference on. IEEE, 2014, pp. 1229-1232.

[3] Satellite Earth Stations and Systems, "Harmonised EN for earth stations on mobile platforms (ESOMP) transmitting towards satellites in geostationary orbit in the $27,5 \mathrm{GHz}$ to $30,0 \mathrm{GHz}$ frequency bands covering essential requirements under article 3.2 of the R\&TTE Directive," ETSI Doc no. EN303978.

[4] R. Lenormand, A. Hirsch, P. Martineau, J. I. Herranz-Herruzo, A. ValeroNogueria, P. Vincent, J.-F. David, and L. Laborde, "Directional mobile antenna with polarization switching by displacement of radiating panels," Feb. 16 2016, US Patent 9,263,801.

[5] K.-S. Son, S.-Y. Hwang, C.-G. Park, and J.-W. Yu, "Waveguide slot array in-motion antenna for receiving both RHCP and LHCP using single layer polarizer," in Microwave Conference, 2006. APMC 2006. Asia-Pacific. IEEE, 2006, pp. 331-334.

[6] R. Lenormand, A. Hirsch, J.-L. Almeida, A. Valero-Nogueira, J. I. Herranz-Herruzo, and D. Renaud, "Compact switchable RHCP/LHCP mobile Ka-band antenna," in Antenna Technology and Applied Electromagnetics (ANTEM), 2012 15th International Symposium on. IEEE, 2012, pp. 1-6.

[7] M. Ferrando-Rocher, J. I. Herranz-Herruzo, A. Valero-Nogueira, and V. M. Rodrigo, "Circularly polarized slotted waveguide array with improved axial ratio performance," IEEE Transactions on Antennas and Propagation, vol. 64, no. 9, pp. 4144-4148, 2016.

[8] E. Rajo-Iglesias and P.-S. Kildal, "Groove gap waveguide: A rectangular waveguide between contactless metal plates enabled by parallel-plate cut-off," in Antennas and Propagation (EuCAP), 2010 Proceedings of the Fourth European Conference on. IEEE, 2010, pp. 1-4.

[9] J. Herranz, M. Baquero, A. Valero-Nogueira, D. Sánchez, and J. Balbastre, "Optimized design of slotted waveguide arrays loaded with parasitic dipoles for circular polarization at Ka band," in Antennas and Propagation, 2009. EuCAP 2009. 3rd European Conference on. IEEE, 2009, pp. 3487-3491.

[10] CST, "Computer simulation technology: Microwave studio," Computer Simulation Technology Std, 2011.

[11] J. I. Herranz Herruzo, A. Valero-Nogueira, S. M. Giner, and A. V. Jiménez, "Untilted narrow-wall slots excited by parasitic dipoles in groove gap waveguide technology," IEEE Transactions on Antennas and Propagation, vol. 63, no. 11, pp. 4759-4765, 2015. 